\title{
Connective tissue growth factor stimulates the proliferation, migration and differentiation of lung fibroblasts during paraquat-induced pulmonary fibrosis
}

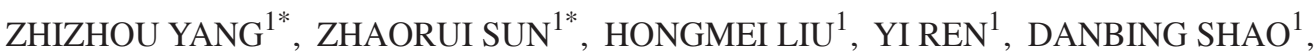 \\ WEI ZHANG ${ }^{1}$, JINFENG LIN ${ }^{1}$, JOY WOLFRAM ${ }^{2}$, FENG WANG ${ }^{3}$ and SHINAN NIE ${ }^{1}$
}

\begin{abstract}
${ }^{1}$ Department of Emergency Medicine, Jinling Hospital, Medical School of Nanjing University, Nanjing, Jiangsu 210002;
${ }^{2}$ CAS Key Laboratory for Biomedial Effects of Nanomaterials \& Nanosafety, National Center for Nanoscience and Technology of China, University of Chinese Academy of Sciences, Beijing 100190; ${ }^{3}$ Department of Gastroenterology, The Tenth People's Hospital of Shanghai, Tongji University, Shanghai 200072, P.R. China
\end{abstract}

Received July 10, 2014; Accepted March 9, 2015

DOI: $10.3892 / \mathrm{mmr} .2015 .3537$

\begin{abstract}
It is well established that paraquat (PQ) poisoning can cause severe lung injury during the early stages of exposure, finally leading to irreversible pulmonary fibrosis. Connective tissue growth factor (CTGF) is an essential growth factor that is involved in tissue repair and pulmonary fibrogenesis. In the present study, the role of CTGF was examined in a rat model of pulmonary fibrosis induced by PQ poisoning. Histological examination revealed interstitial edema and extensive cellular thickening of interalveolar septa at the early stages of poisoning. At 2 weeks after PQ administration, lung tissue sections exhibited a marked thickening of the alveolar walls with an accumulation of interstitial cells with a fibroblastic appearance. Masson's trichrome staining revealed a patchy distribution of collagen deposition, indicating pulmonary fibrogenesis. Western blot analysis and immunohistochemical staining of tissue samples demonstrated that CTGF expression was significantly upregulated in the PQ-treated group. Similarly, PQ treatment of MRC-5 human lung fibroblast cells caused an increase in CTGF in a dose-dependent manner. Furthermore, the addition of CTGF to MRC-5 cells triggered cellular proliferation and migration. In addition, CTGF induced the differentiation of fibroblasts to myofibroblasts, as was evident from increased expression of $\alpha$-smooth muscle actin ( $\alpha$-SMA) and collagen. These findings demonstrate that PQ causes increased CTGF expression, which triggers
\end{abstract}

Correspondence to: Professor Shinan Nie, Department of Emergency Medicine, Jinling Hospital, Medical School of Nanjing University, 305 Zhongshan East Road, Nanjing, Jiangsu 210002, P.R. China E-mail: shn_nie@sina.com

*Contributed equally

Key words: paraquat, pulmonary fibrosis, connective tissue growth factor, myofibroblast differentiation proliferation, migration and differentiation of lung fibroblasts. Therefore, CTGF may be important in PQ-induced pulmonary fibrogenesis, rendering this growth factor a potential pharmacological target for reducing lung injury.

\section{Introduction}

Paraquat dichloride (1,1'-dimethyl-4,4'-bipyridinium dichloride; methyl viologen; PQ) is an effective and widely used herbicide that can cause pulmonary fibrosis $(1,2)$. Previous studies have demonstrated that the lungs are one of the primary target organs for PQ-induced toxicity in humans and animals $(1,3)$. The acute toxic effects of PQ, including pulmonary edema and hypoxia, lead to irreversible pulmonary fibrosis. Notably, $1,000 \mathrm{~s}$ of mortalities due to intentional or accidental ingestion of PQ have been reported $(4,5)$. Although certain drugs, including glucocorticoids, antioxidants and cytotoxic drugs, can mitigate toxicity (6-9), the treatment of acute PQ poisoning is often poor and mortality rates remain high $(10,11)$. Since an effective PQ antidote has yet to be identified, studies on the molecular mechanisms of PQ-induced pulmonary fibrosis are critical for improving treatment and reducing mortality.

Connective tissue growth factor (CTGF) is a cysteine-rich extracellular matrix-associated heparin-binding protein that belongs to the CCN family $(12,13)$. CTGF is widely expressed in numerous tissues at low levels, however, is markedly upregulated in fibrotic and cancerous tissue (14). CTGF regulates various biological processes associated with fibrogenesis, including cellular adhesion, proliferation, migration, differentiation, extracellular matrix (ECM) production (15-17) and angiogenesis (18). In particular, CTGF has been found to promote deposition of several ECM proteins, including collagen, fibronectin and tenascin C $(19,20)$. Aberrant ECM production by lung fibroblasts has been associated with fibrosis in several models of pulmonary injury $(21,22)$. ECM production could further be stimulated as a consequence of lung fibroblast proliferation, migration and differentiation into myofibroblasts (23). Accordingly, CTGF has been demonstrated 
to affect various cell types involved in the fibrogenic process, including type II alveolar epithelial cells, endothelial cells, mesenchymal stem cells and lung fibroblasts (16).

Although CTGF has been demonstrated to be important in pulmonary fibrosis induced by bleomycin and hyperoxia $(24,25)$, an association between this growth factor and PQ-induced lung injury has not been established. In particular, the effect of CTGF on ECM production, cellular proliferation, migration and myofibroblast differentiation in association with $\mathrm{PQ}$ poisoning remains to be elucidated. The aim of the present study was to investigate the effects of PQ on CTGF expression and subsequent pulmonary fibrosis.

\section{Materials and methods}

Ethics statement. All animals were handled in accordance with guidelines approved by the Experimentation Ethics Review Committee of Nanjing University (Nanjing, China). The rats were fed with commercial rat chow, provided with water ad libitum and kept on a 12:12 h light-dark cycle.

Cell culture. MRC-5 lung fibroblasts (human lung fibroblasts; American Type Culture Collection, Manassas, VA, USA; cat. no. CCL 171) were cultured in high Dulbecco's modified Eagle's medium (DMEM; HyClone Laboratories, Inc., Logan, UT, USA) with $10 \%$ fetal bovine serum (FBS; Invitrogen Life Technologies, Carlsbad, CA, USA) supplemented with $1 \% \mathrm{~L}$-glutamine and $1 \%$ penicillin/streptomycin solution. Cells were incubated at $37^{\circ} \mathrm{C}$ in $5 \% \mathrm{CO}_{2}$ and routinely passaged upon reaching $80 \%$ confluency, using $0.25 \%$ trypsin and a $1: 3$ cell dilution for each passage.

Cell viability. The viability of lung fibroblasts was evaluated using a Cell Counting kit-8 (CCK-8; Dojindo Laboratories, Kumamoto, Japan) assay. Cells were plated in 6-well plates at a density of $2 \times 10^{6}$ cells $/ \mathrm{ml}$ for $12 \mathrm{~h}$ and treated with various concentrations of CTGF (50-200 ng/ml; PeproTech, Inc., Rocky Hill, NJ, USA) for 24, 48 or $72 \mathrm{~h}$. The cells were then transferred into a 96-well culture plate $(\mathrm{n}=8)$ at a density of $2 \times 10^{4}$ cells $/ 100 \mu 1 /$ well for $12 \mathrm{~h}$. The culture medium was removed and $100 \mu \mathrm{l}$ of serum-free medium containing $10 \mu \mathrm{l}$ of CCK- 8 solution was added to each well. After a $4 \mathrm{~h}$ incubation period, the absorbance was measured at an optical density (OD) of $450 \mathrm{~nm}$ using a multi-detection microplate reader (VersaMax; Molecular Devices, Sunnyvale, CA, USA).

Cell migration. A double-chamber system was used to perform a transwell migration assay to determine the migration ability of lung fibroblasts. Cells were seeded in a 6-well plate with serum-free medium at a density of $4 \times 10^{4}$ cells/per upper chamber. Culture medium (1 ml) with $10 \%$ FBS containing various concentrations of CTGF $(50-200 \mathrm{ng} / \mathrm{ml})$ was simultaneously added to the lower chamber. The upper and lower chambers were separated by a permeable polycarbonate membrane with a pore size of $8 \mu \mathrm{m}$. The cells were permitted to migrate to the lower chamber for $24 \mathrm{~h}$. Following this incubation period, cells that had entered the lower surface of the filter membrane were fixed with a cold solution of $4 \%$ paraformaldehyde for $30 \mathrm{~min}$. Cells were then washed three times with phosphate-buffered saline (PBS) and stained with $0.1 \%$ crystal violet for $30 \mathrm{~min}$. Cells that remained in the upper surface were gently scraped off with a cotton swab. A total of 10 different fields of view from each membrane were randomly selected and captured using a photomicroscope (BX51; Olympus, Tokyo, Japan). Cell migration was quantified by counting the number of migrated cells. Experiments were performed three times in duplicate.

Animal groups and experimental protocol. A total of 48 adult male Sprague-Dawley (SD) rats weighing 200-250 g were purchased from the Animal Center of Nanjing University (Nanjing, China). The SD rats were randomly divided into two groups ( $\mathrm{n}=24$ for each group). The experimental group received a single intraperitoneal injection of $\mathrm{PQ}(30 \mathrm{mg} / \mathrm{kg})$, while the control group received an equivalent volume of sterile saline. The rats were then sacrificed by cervical dislocation after 7, 14 and 28 days.

Histological analysis. Following sacrificing the animals, the lungs were removed and the left lung was fixed with $4 \%$ paraformaldehyde for $16 \mathrm{~h}$. The tissue samples were then processed using graded alcohol, xylene and paraffin and blocked in paraffin. The paraffin-embedded sections (5 $\mu \mathrm{m}$ thick) were stained using a hematoxylin and eosin (H\&E) kit (Biyuntian, Inc., Nantong, China) and a Masson's trichrome staining kit (Nanjing Jiancheng Bioengineering Institute, Nanjing, China), according to the manufacturer's instructions. The slides with $\mathrm{H} \& \mathrm{E}$ staining were examined using light microscopy (Eclipse TE2000-S; Nikon Corporation, Tokyo, Japan) and images were captured to determine the integrity of the tissue. The severity of pulmonary fibrosis in the lung sections stained for collagen with Masson's trichrome stain was determined by a histopathologist who was blinded to the protocol design.

Immunohistochemical staining. Formalin-fixed, paraffin-embedded skin sections were stained with rabbit polyclonal anti-CTGF antibodies (cat. no. ab6992; Abcam, Inc., Cambridge, MA, USA). Following transferal through a graded series of alcohol and xylene, lung tissue samples were embedded in paraffin and sectioned $(5 \mu \mathrm{m})$. The sample sections were mounted onto poly-L-lysine-coated slides and processed for immunohistochemical analysis, according to a similar procedure as previously reported (26). Briefly, sections were incubated overnight at room temperature with $3 \%$ bovine serum albumin (BSA) for $30 \mathrm{~min}$ at $37^{\circ} \mathrm{C}$ for blockade of non-specific binding sites and then incubated overnight at $4^{\circ} \mathrm{C}$ with the primary antibody targeting CTGF (1:200 dilution). Specificity of the antibody was examined using normal rabbit serum instead of the primary antibody. The slides were then incubated with a secondary biotinylated goat anti-rabbit antibody (1:200 dilution; cat. no. BA1003; Wuhan Boster Biological Technology, Ltd., Wuhan, China) for $30 \mathrm{~min}$ at $37^{\circ} \mathrm{C}$. Following rinsing, the slides were incubated with horseradish peroxidase-conjugated streptavidin and then washed with deionized water. The samples were then exposed to 3,3'-diaminobenzidine substrate solution for $10 \mathrm{~min}$, counterstained with hematoxylin and mounted with coverslips. Images were captured on a Nikon Eclipse TE2000-S microscope (Nikon Corporation). A brown reaction product was considered a positive result. 
Immunofluorescence. To evaluate the effect of CTGF on myofibroblast differentiation of human lung fibroblasts, MRC-5 lung fibroblasts were treated with $100 \mathrm{ng} / \mathrm{ml}$ CTGF (PeproTech, Inc.) for 3 days. Immunofluorescence staining was performed as previously described (21). Briefly, MRC-5 cells were fixed with $4 \%$ paraformaldehyde and permeabilized with $0.1 \%$ Triton X-100 for $10 \mathrm{~min}$. The cells were then incubated with PBS containing $2 \% \mathrm{BSA}$ for $1 \mathrm{~h}$ at $37^{\circ} \mathrm{C}$ to block unspecific binding sites. The fixed cells were then incubated with the following primary antibodies: Rabbit polyclonal anti- $\alpha$-smooth muscle actin ( $\alpha$-SMA; 1:200 dilution; cat. no. ab5694) and rabbit polyclonal anti-collagen I (1:200 dilution; cat. no. ab34710; Abcam, Inc., Cambridge, MA, USA) at $4^{\circ} \mathrm{C}$ for $16 \mathrm{~h}$. Following three washes with PBS, cells were incubated with a secondary antibody (1:400 dilution; goat anti-rabbit Alexa Fluor 594 or 488; Invitrogen Life Science, Gaithersburg, MD, USA) in $2 \% \mathrm{BSA}$ for $1 \mathrm{~h}$ at $37^{\circ} \mathrm{C}$ in the dark. Nuclear staining was performed using $5 \mathrm{mg} / \mathrm{ml}$ 4',6-diamidino-2-phenylindole (Biyuntian, Inc.). Cells were visualized using a confocal fluorescence microscope (Fluoview FV10i; Olympus).

Western blot analysis. In order to investigate the effects of PQ on protein levels of CTGF, MRC-5 cells were treated with various concentrations of PQ (50-500 $\mu \mathrm{M}$; Sigma-Aldrich, St. Louis, MO, USA) for $72 \mathrm{~h}$. Protein samples were obtained from cultured cells and animals (right lung, $n=8$ ) treated with PQ. Western blot analysis of protein lysates was performed as previously described (21). Briefly, cells and lung tissues were lysed in ice-cold RIPA extraction buffer $(150 \mathrm{mM}$ $\mathrm{NaCl}, 10$ mM Tris-HCl, pH 7.4, 1\% Triton X-100, 1\% sodium deoxycholate and $0.1 \%$ SDS) containing a protease inhibitor cocktail (Roche Diagnostics, Indianapolis, IN, USA) for $30 \mathrm{~min}$. The whole lysates were then centrifuged at $12,000 \mathrm{x} \mathrm{g}$ for $30 \mathrm{~min}$ and the protein concentration in the supernatant was determined using a bicinchoninic acid assay (Wuhan Boster Biological Technology, Ltd.). The protein samples were boiled for $10 \mathrm{~min}$ and $20 \mu \mathrm{l}$ aliquots were then subjected to $12 \%$ SDS-polyacrylamide gel electrophoresis. The protein bands were electrophoretically transferred onto a polyvinylidene fluoride membrane, which was incubated in blocking buffer (1X PBS, $0.1 \%$ Tween-20, 1\% BSA and 5\% non-fat milk) for $1 \mathrm{~h}$ at $37^{\circ} \mathrm{C}$. The membrane was then exposed to primary antibodies (at a 1:3,000 dilution) against rabbit $\alpha$-SMA, rabbit collagen I and mouse $\beta$-actin (Abcam, Inc.) in blocking buffer overnight at $4^{\circ} \mathrm{C}$. Following three washes in PBS containing $0.05 \%$ Tween 20 , the membrane was incubated with the secondary antibody (horseradish peroxidase-conjugated goat anti-rabbit/mouse IgG; Wuhan Boster Biological Technology, Ltd.) at $37^{\circ} \mathrm{C}$ for $1 \mathrm{~h}$. Visualization of immunoreactive protein bands was performed with an enhanced chemiluminescence detection kit (Amersham Biosciences, Piscataway, NJ, USA) using an Odyssey Scanning System (LI-COR, Inc., Lincoln, NE, USA).

Hydroxyproline (HP) content. The quantity of collagen in the lung tissue was determined by analysis of HP content according to the manufacturer's instructions of the detection kit (Nanjing Jiancheng Bioengineering Institute). The absorbance was measured at $550 \mathrm{~nm}$ and the HP content was determined using a standard curve $(0-100 \mathrm{mg} / \mathrm{ml})$.

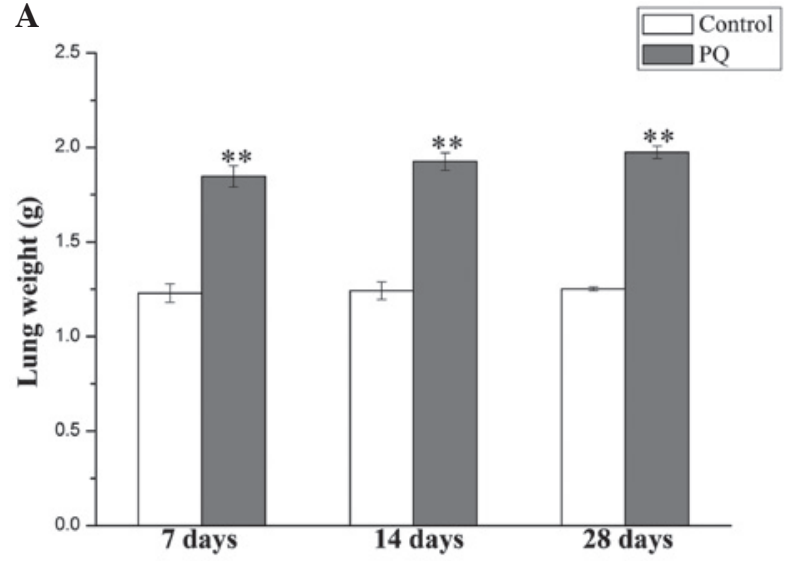

B
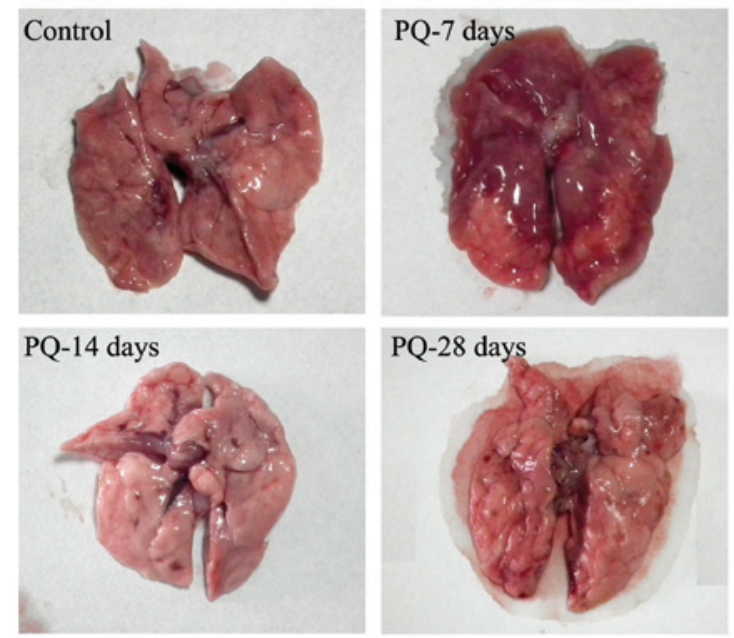

Figure 1. PQ-induced lung injury and pulmonary fibrosis. (A) Weights of lung tissue in the control and PQ-treated mice 7, 14 and 28 days after PQ administration. Data are expressed as the mean \pm standard deviation. ${ }^{* *} \mathrm{P}<0.01$ vs. the control group $(n=8)$. (B) Representative images of whole lungs 7, 14 and 28 days after PQ administration. PQ, paraquat.

Statistical analysis. Data are expressed as the mean \pm standard deviation. Differences among groups were evaluated by one-way analysis of variance using Statistical Package for the Social Sciences (SPSS) version 18.0 software (SPSS, Inc., Chicago IL, USA). $\mathrm{P}<0.05$ was considered to indicate a statistically significant difference.

\section{Results}

$P Q$-induced lung injury and pulmonary fibrosis. After PQ administration for 7, 14 and 28 days, rats were sacrificed and the lungs were collected. As shown in Fig. 1A, the weight of the lungs increased in response to PQ administration, indicating the presence of pulmonary inflammation and fibrosis. In addition, the macroscopic appearance of the lungs was fibrotic (Fig. 1B). These results suggest that PQ is able to induce lung injury, inflammation and fibrosis in this rat model.

Histopathological analyses of rat lungs treated with $P Q$. To investigate the effects of PQ on lung tissue, serial lung sections from PQ-treated rats (7, 14 and 28 days after PQ administration) were stained with H\&E or Masson's trichrome and examined 
A

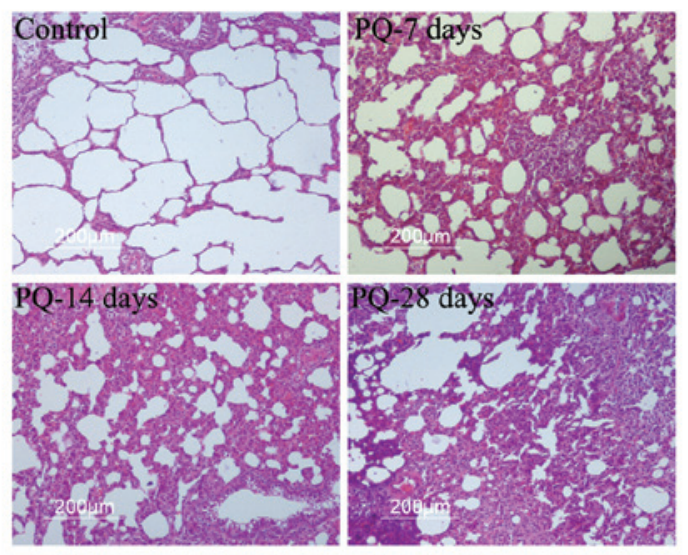

B

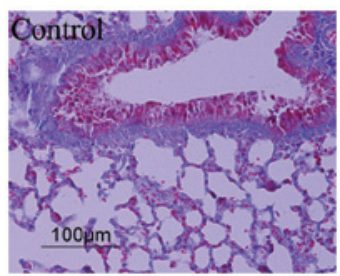

C

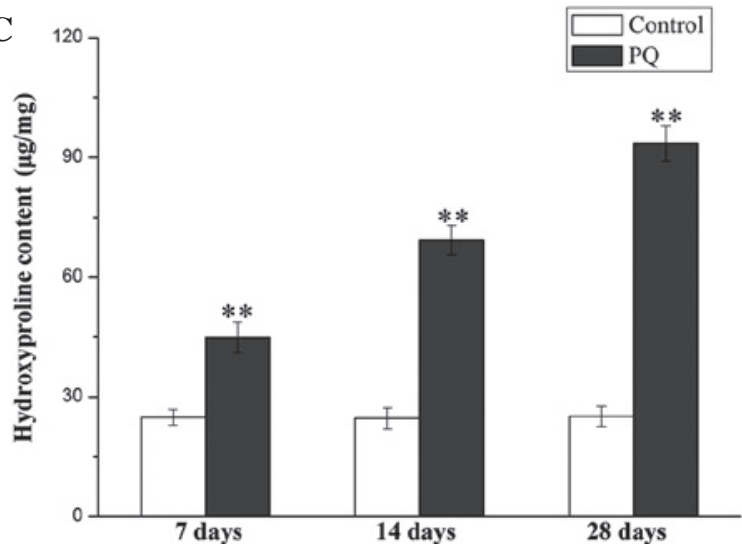

Figure 2. PQ-induced pulmonary fibrogenesis and collagen deposition in rat lung tissue. (A) Histopathological views of lung tissues from rats following PQ exposure (7, 14 and 28 days). Lung sections were stained with hematoxylin and eosin. (B) Masson's trichrome staining analysis of collagen (blue color) on day 28 following PQ exposure. (C) Hydroxyproline content in rat lungs on day 7, 14 and 28 following PQ administration. All data are presented as the mean \pm standard deviation. ${ }^{* *} \mathrm{P}<0.01$ vs. the control group $(\mathrm{n}=8)$. $\mathrm{PQ}$, paraquat.

using a light microscope. Representative histological sections from lungs of rats in each experimental group are shown in Fig. 2A. Lung tissues in the control group appeared histologically normal, showing no signs of inflammation or epithelial damage. By contrast, histopathological examination of the lung tissue following PQ administration revealed alterations in the lung structure. After 7 days, the lungs exhibited marked alterations in tissue structure, exhibiting signs of acute injury with interstitial edema and widespread inflammatory cell infiltration in the alveolar space and septum. In addition, an infiltration of mononuclear inflammatory cells, fibroblast proliferation, extensive cellular thickening and fibrosis was evident after 14 days. Similarly, at day 28, H\&E staining indicated thickened alveolar walls and pulmonary interstitial fibrosis. Masson's trichrome staining revealed that collagen deposition (blue staining) in the PQ group was increased compared with the control group (Fig. 2B). In conclusion, histological examination demonstrated that $\mathrm{PQ}$ administration induced lung injury and pulmonary fibrosis.

$H P$ content in the lungs following $P Q$ administration. In order to evaluate collagen deposition in the lung tissues, the HP content was determined 7, 14 and 28 days after PQ administration. As shown in Fig. 2C, PQ caused a statistically significant $(\mathrm{P}<0.05)$ increase in the amount of $\mathrm{HP}$ at all time points. On day 28, the HP content in the PQ group was more than three times higher than in the control group. The results of HP quantification were consistent with the histopathological results.

$P Q$ induces expression of CTGF in lung tissues and lung fibroblasts. Immunohistochemical staining was used to detect the expression of CTGF in lung tissue. As shown in Fig. 3A, the CTGF expression was significantly increased 28 days after $\mathrm{PQ}$ administration $(\mathrm{P}<0.01)$. Western blot analysis was also performed to confirm that the CTGF expression was enhanced in response to PQ. As shown in Fig. 3B, PQ-induced a time-dependent increase in CTGF protein levels. Furthermore, western blot analysis was performed using MRC- 5 cell lysates to evaluate whether the same trend could be observed in vitro. Following exposure to various concentrations of PQ (50-500 $\mu \mathrm{M})$, the results indicated that the cells exhibited significantly higher levels of CTGF protein $(\mathrm{P}<0.01$; Fig. $3 \mathrm{C})$. The results suggest that PQ-induced CTGF expression may contribute to pulmonary fibrogenesis.

CTGF promotes the proliferation and migration of lung fibroblasts. The effect of CTGF on the proliferation and migration of lung fibroblasts was evaluated, as these biological processes are important in pulmonary fibrogenesis. As shown in Fig. 4A, the cell viability was significantly increased in response to CTGF $(\mathrm{P}<0.01)$. The data indicate that CTGF promotes cell proliferation in a dose- and time-dependent manner. As shown in Figs. $4 \mathrm{~B}$ and $\mathrm{C}$, the results from the transwell migration assay reveal that CTGF improves lung fibroblast migration in a statistically significant manner $(\mathrm{P}<0.01)$. In particular, $200 \mathrm{ng} / \mathrm{ml}$ of CTGF caused a 3.6-fold increase in cell migratory capacity. These results highlight the possibility that CTGF is able to mediate PQ-induced pulmonary fibrogenesis.

CTGF induces myofibroblast differentiation of lung fibroblasts. To further investigate the role of CTGF in pulmonary fibrogenesis, the differentiation of lung fibroblasts into myofibroblasts was examined. MRC-5 cells were incubated with $100 \mathrm{ng} / \mathrm{ml}$ of CTGF for 3 days. Immunofluorescence and western blot analysis were used to measure expression levels of $\alpha$-SMA 

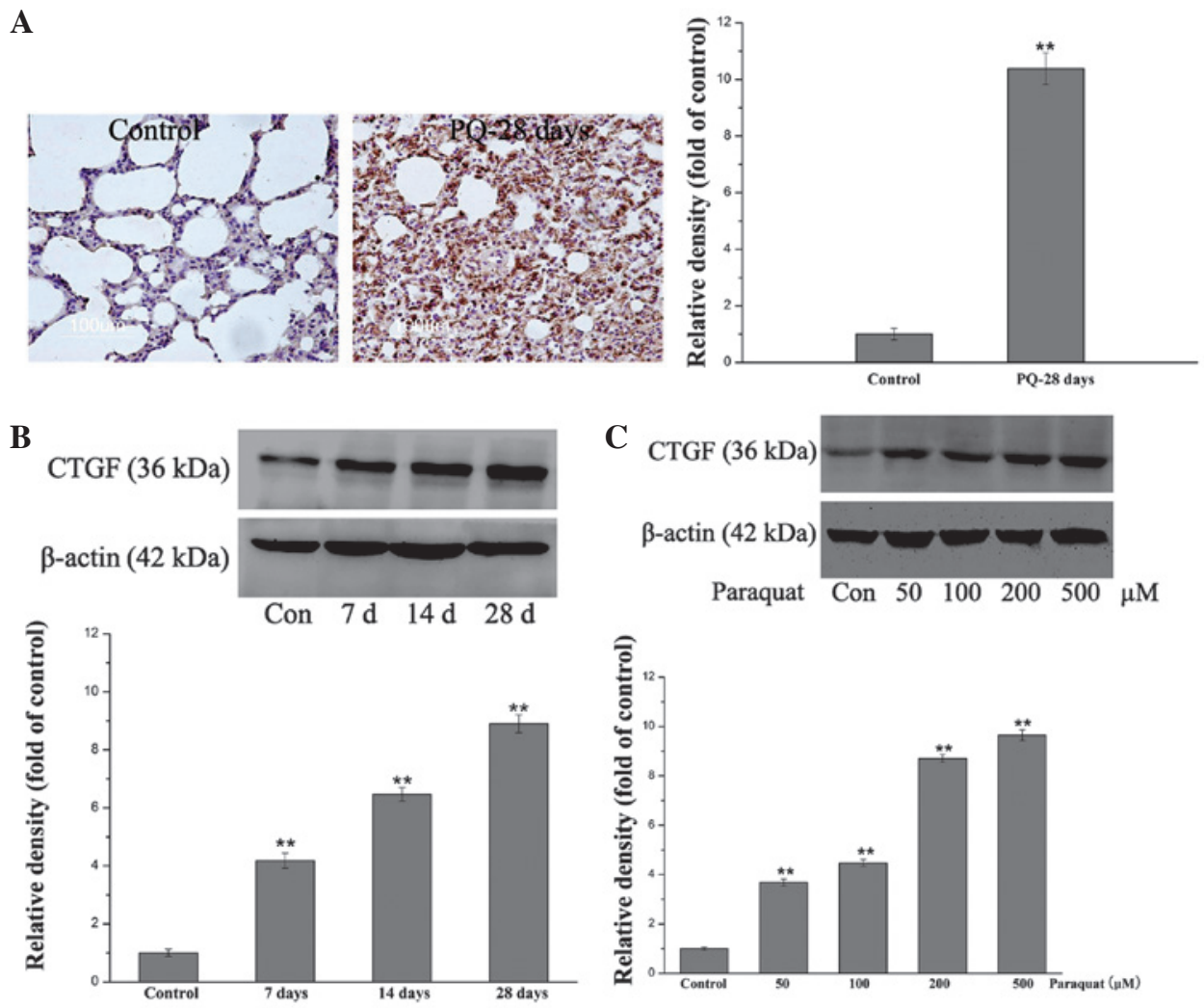

Figure 3. PQ-induced CTGF expression in rat lungs and MRC-5 human lung fibroblast cells. (A) Immunohistochemistry of CTGF in lung tissue 28 days after $\mathrm{PQ}$ administration. (B) Western blot analysis of CTGF in lung tissues 7, 14 and 28 days after PQ exposure $(\mathrm{P}<0.01)$. (C) Western blot analysis of CTGF in MRC-5 cells exposed to 50,100, 200 and $500 \mu \mathrm{M}$ of PQ. Western blot bands were quantified using densitometry analysis and normalized to the expression of $\beta$-actin. Densitometry data are presented as the mean \pm standard deviation. ${ }^{* *} \mathrm{P}<0.01$ vs. the control group $(\mathrm{n}=6)$ for each experiment. $\mathrm{PQ}$, paraquat; $\mathrm{CTGF}$, connective tissue growth factor.

A

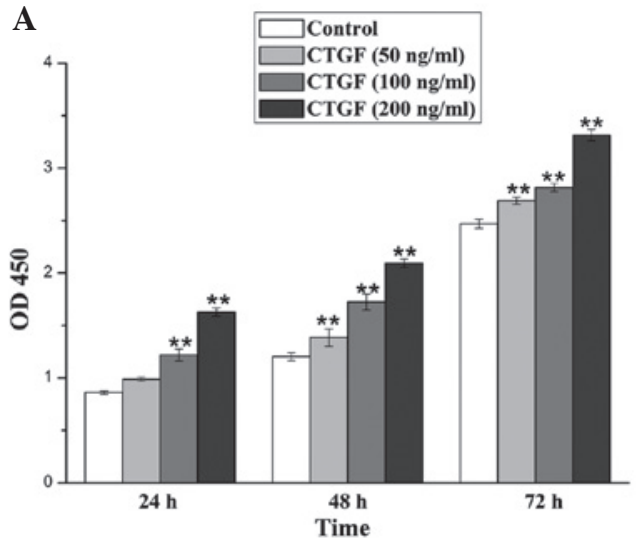

C

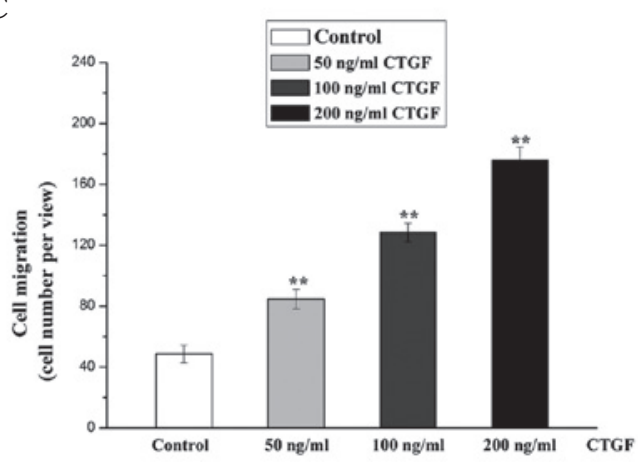

B

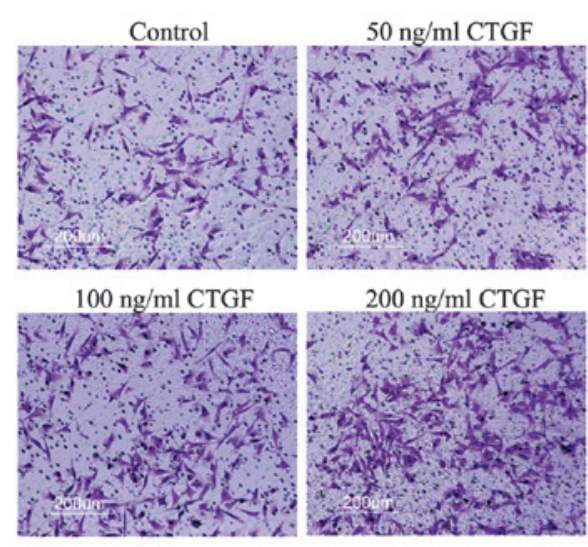

Figure 4. CTGF-induced proliferation and migration of lung fibroblasts. (A) Cell proliferation of MRC-5 cells exposed to 50, $100 \mathrm{and} 200 \mathrm{ng} / \mathrm{ml}$ of CTGF $(\mathrm{n}=6)$. (B) Transwell migration analysis of MRC-5 cells treated with 50, 100 and $200 \mathrm{ng} / \mathrm{ml}$ of CTGF. The images are representative of three independent experiments. (C) Quantitative presentation of cell migration ability. The data are presented as the mean \pm standard deviation from three experiments. ${ }^{* *} \mathrm{P}<0.01$, vs. the control group. $\mathrm{PQ}$, paraquat; $\mathrm{CTGF}$, connective tissue growth factor. 


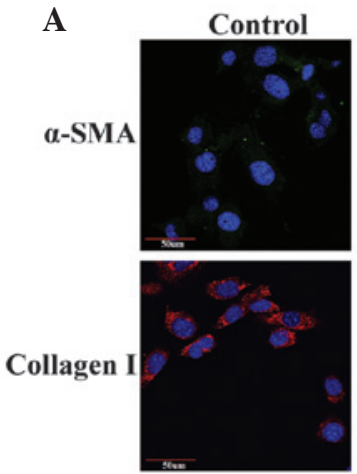

C

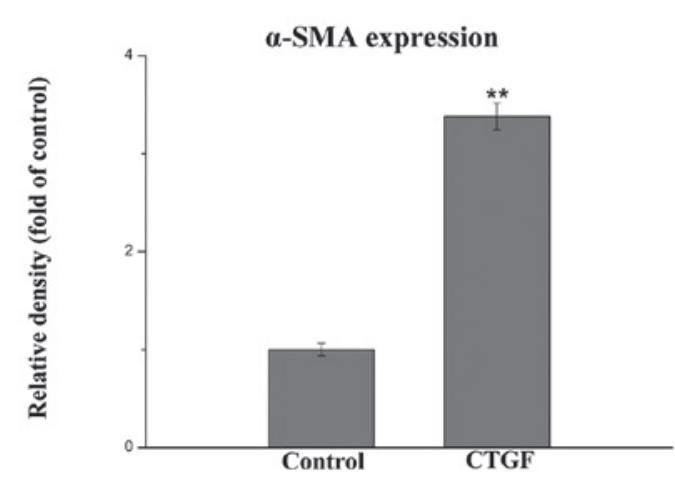

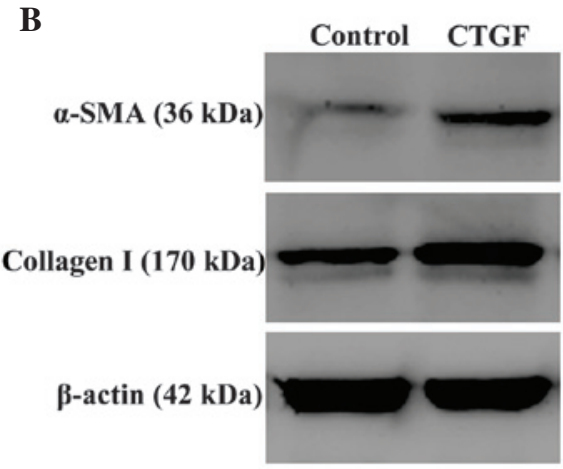

D

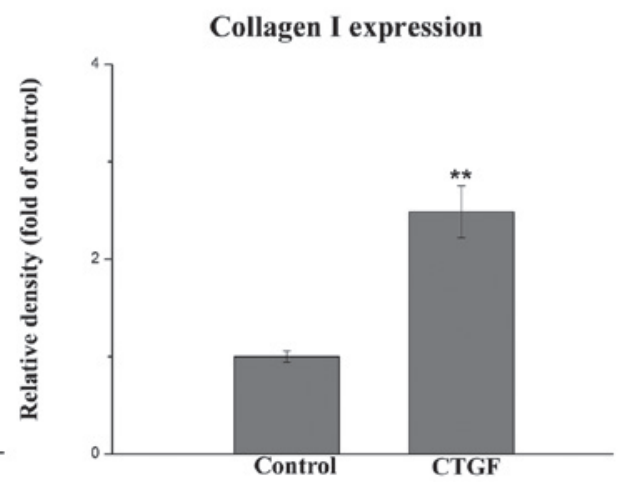

Figure 5. CTGF-induced differentiation of MRC-5 lung fibroblasts into myofibroblasts. (A) Immunofluorescence analysis of $\alpha-S M A$ and collagen I in MRC-5 fibroblasts following treatment with CTGF for 3 days. Magnification, x600 (scale bar=50 $\mu \mathrm{m}$ ). (B) Western blot analysis of $\alpha$-SMA and collagen I in whole cell lysates. $\beta$-actin was used as a loading control. Quantitative analysis of western blot results of (C) CTGF-induced $\alpha$-SMA expression and (D) collagen I expression. Protein bands were quantified by densitometry and normalized to the expression of $\beta$-actin. Densitometry data are presented as the mean \pm standard deviation. ${ }^{* *} \mathrm{P}<0.01$ vs. the control group. $\mathrm{n}=6$ for each group. $\alpha$-SMA, $\alpha$-smooth muscle actin; CTGF, connective tissue growth factor.

and collagen I, which are myofibrolast differentiation markers. As shown in Fig. 5A, immunofluorescence staining indicated that $\alpha$-SMA (stained green) and collagen I (stained red) protein expression was increased in the CTGF-induced group, as compared with the control group. In addition, as shown in Figs. 5B-D, western blotting demonstrated that CTGF induced a pronounced increase in the protein expression levels of $\alpha$-SMA and collagen I, indicating the differentiation of lung fibroblasts into myofibroblasts. This differentiation process has previously been associated with lung fibrosis (27).

\section{Discussion}

PQ has previously been found to cause acute lung injury and pulmonary fibrosis with interstitial collagen deposition, which leads to reduced functional capacity (1). PQ poisoning is a severe health problem, as numerous human mortalities have occurred as a consequence of PQ ingestion $(4,5)$. The lung is the major target organ for this toxic agent, as alveoli type II epithelial cells absorb PQ through an active polyamine uptake process (28-30). PQ can accumulate in lung tissue and reach peak plasma concentrations within $2 \mathrm{~h}$ after ingestion (31). Notably, the concentration of PQ in the lung parenchyma can be 10-20 times higher than that in the plasma (32). The signaling pathways that lead to PQ-induced pulmonary fibrosis remain to be elucidated. Previous studies have focused on clarifying the molecular mechanisms of PQ poisoning to determine useful molecular targets for developing therapeutic strategies. The present study examined the role of CTGF in PQ-induced collagen production and myofibroblast differentiation of human lung fibroblasts.

CTGF is a downstream cooperative mediator of the transforming growth factor- $\beta$ signaling pathway and is widely expressed in numerous tissues at low physiological levels. However, this growth factor is markedly upregulated at the pathological sites of numerous animal models of human disease, including pulmonary fibrosis, liver fibrosis, skin fibrosis, cancer and various types of malignancy $(14,33,34)$. In particular, increased levels of CTGF have previously been reported in patients with severe pulmonary fibrosis and animal models of pulmonary fibrosis (25). In the present study, PQ exposure caused alterations in lung architecture, which was evident from interstitial edema, extensive cellular thickening of interalveolar septa, increased interstitial cells with a fibroblastic appearance and excessive collagen deposition. Concurrently, it was found that PQ exposure induces CTGF expression in vitro and in vivo. A previous in vitro study also indicated that CTGF can exert an effect on a number of cell types, thereby promoting biological processes associated with fibrogenesis, including cell proliferation, migration and ECM production (16). The present study demonstrated that CTGF can induce the proliferation and migration of MRC-5 lung fibroblasts. Therefore, it is likely that CTGF is a mediator of PQ-induced pulmonary fibrogenesis.

Accumulating evidence suggests that the overexpression of collagen and $\alpha$-SMA in lung fibrotic lesions is associated with fibrotic lung disease (35). Collagen is the major ECM component of the lungs and increases at the early stages of acute lung injury, affecting respiratory mechanics. Myofibroblasts that 
originate from the differentiation of lung fibroblasts exhibit morphological and biochemical characteristics of lung fibroblasts and smooth muscle cells, which have been considered the main source of ECM within the impaired lungs of patients with idiopathic pulmonary fibrosis $(36,37)$. The results from the present study are supported by a previous study demonstrating that CTGF knockdown animals have a reduced pathogenic fibrotic response (29). The mechanism responsible for protection against fibrosis in CTGF knockdown cells was found to be reduced collagen synthesis (38). In the present study, the results indicate that PQ exposure causes increased levels of collagen and CTGF, in a time-dependent manner. Immunofluorescent staining and western blot analysis reveal that lung fibroblasts treated with CTGF have increased expression of collagen and $\alpha$-SMA. This observation suggests that CTGF can induce myofibroblast differentiation.

In conclusion, our observations imply that PQ-induced overexpression of CTGF may be responsible for pulmonary fibrosis, through promoting the proliferation, migration and myofibroblast differentiation of lung fibroblasts. The present study supports the theory that pharmacological inhibition of CTGF is a feasible strategy to reduce the magnitude of pulmonary fibrosis induced by $\mathrm{PQ}$.

\section{Acknowledgements}

This study was supported by grants from the National Natural Science Foundation of China (grant no. 81401583), the Major Projects Foundation of Nanjing Military Region (grant no. 12Z32), the Medical Science Foundation for Young Cultivation Project of PLA (grant no. 13QNP038) and the Natural Science Foundation of Jinling Hospital (grant nos. 2013023 and 2014004).

\section{References}

1. Dinis-Oliveira RJ, Duarte JA, Sánchez-Navarro A, et al: Paraquat poisonings: mechanisms of lung toxicity, clinical features, and treatment. Crit Rev Toxicol 38: 13-71, 2008.

2. Neves FF, Sousa RB, Pazin-Filho A, et al: Severe paraquat poisoning: clinical and radiological findings in a survivor. J Bras Pneumol 36: 513-516, 2010.

3. Chen CM and Lua AC: Lung toxicity of paraquat in the rat. J Toxicol Environ Health A 60: 477-487, 2000.

4. Baltazar T, Dinis-Oliveira RJ, Duarte JA, et al: Paraquat research: do recent advances in limiting its toxicity make its use safer? $\mathrm{Br}$ J Pharmacol 168: 44-45, 2013.

5. Novaes RD, Gonçalves RV, Cupertino MC, et al: Bark extract of Bathysa cuspidata attenuates extra-pulmonary acute lung injury induced by paraquat and reduces mortality in rats. Int J Exp Pathol 93: 225-233, 2012.

6. Li LR, Sydenham E, Chaudhary B and You C: Glucocorticoid with cyclophosphamide for paraquat-induced lung fibrosis. Cochrane Database Syst Rev 16: CD008084, 2010.

7. Lin JL, Lin-Tan DT, Chen KH, et al: Improved survival in severe paraquat poisoning with repeated pulse therapy of cyclophosphamide and steroids. Intensive Care Med 37: 1006-1013, 2011.

8. Moon JM and Chun BJ: The efficacy of high doses of vitamin C in patients with paraquat poisoning. Hum Exp Toxicol 30: 844-850, 2011.

9. Suntres ZE: Role of antioxidants in paraquat toxicity. Toxicology 180: 65-77, 2002.

10. Koo JR, Kim JC, Yoon JW, et al: Failure of continuous venovenous hemofiltration to prevent death in paraquat poisoning. Am J Kidney Dis 39: 55-59, 2002.

11. Koo JR, Yoon JW, Han SJ, et al: Rapid analysis of plasma paraquat using sodium dithionite as a predictor of outcome in acute paraquat poisoning. Am J Med Sci 338: 373-377, 2009.
12. Harlow CR and Hillier SG: Connective tissue growth factor in the ovarian paracrine system. Mol Cell Endocrinol 187: 23-27, 2002.

13. Takigawa M, Nakanishi T, Kubota S and Nishida T: Role of CTGF/HCS24/ecogenin in skeletal growth control. J Cell Physiol 194: 256-266, 2003.

14. Wang Q, Usinger W, Nichols B, et al: Cooperative interaction of CTGF and TGF- $\beta$ in animal models of fibrotic disease. Fibrogenesis Tissue Repair 4: 4, 2011.

15. Moussad EE and Brigstock DR: Connective tissue growth factor: what's in a name? Mol Genet Metab 71: 276-292, 2000.

16. Ponticos M, Holmes AM, Shi-wen X, et al: Pivotal role of connective tissue growth factor in lung fibrosis: MAPK-dependent transcriptional activation of type I collagen. Arthritis Rheum 60: 2142-2155, 2009.

17. Bogatkevich GS, Ludwicka-Bradley A, Singleton CB, et al: Proteomic analysis of CTGF-activated lung fibroblasts: identification of IQGAP1 as a key player in lung fibroblast migration. Am J Physiol Lung Cell Mol Physiol 295: L603-L611, 2008.

18. Shimo T, Kubota S, Kondo S, et al: Connective tissue growth factor as a major angiogenic agent that is induced by hypoxia in a human breast cancer cell line. Cancer Lett 174: 57-64, 2001.

19. Chujo S, Shirasaki F, Kawara S, et al: Connective tissue growth factor causes persistent proalpha2(I) collagen gene expression induced by transforming growth factor-beta in a mouse fibrosis model. J Cell Physiol 203: 447-456, 2005.

20. Heng EC, Huang Y, Black SA Jr and Trackman PC: CCN2, connective tissue growth factor, stimulates collagen deposition by gingival fibroblasts via module 3 and alpha6- and betal integrins. J Cell Biochem 98: 409-420, 2006.

21. Sun Z, Gong X, Zhu H, et al: Inhibition of Wnt/ $/$-catenin signaling promotes engraftment of mesenchymal stem cells to repair lung injury. J Cell Physiol 229: 213-224, 2014.

22. McAnulty RJ: Fibroblasts and myofibroblasts: their source, function and role in disease. Int J Biochem Cell Biol 39: 666-671, 2007.

23. Scotton CJ and Chambers RC: Molecular targets in pulmonary fibrosis: the myofibroblast in focus. Chest 132: 1311-1321, 2007.

24. Bonniaud P, Margetts PJ, Kolb M, et al: Adenoviral gene transfer of connective tissue growth factor in the lung induces transient fibrosis. Am J Respir Crit Care Med 168: 770-778, 2003.

25. Chen CM, Wang LF, Chou HC,Lang YD and Lai YP: Up-regulation of connective tissue growth factor in hyperoxia-induced lung fibrosis. Pediatr Res 62: 128-133, 2007.

26. Sun Z, Wang C, Shi C, et al: Activated Wnt signaling induces myofibroblast differentiation of mesenchymal stem cells, contributing to pulmonary fibrosis. Int J Mol Med 33: 1097-1109, 2014.

27. Phan SH: The myofibroblast in pulmonary fibrosis. Chest 122 (6 Suppl): 286S-289S, 2002

28. Ahmed AA: Protective effect of montelukast on paraquat-induced lung toxicity in rats. Biosci Trends 3: 63-72, 2009.

29. Tomita M, Okuyama T, Katsuyama H, et al: Mouse model of paraquat-poisoned lungs and its gene expression profile. Toxicology 231: 200-209, 2007.

30. Hoet PH and Nemery B: Polyamines in the lung: polyamine uptake and polyamine-linked pathological or toxicological conditions. Am J Physiol Lung Cell Mol Physiol 278: L417-L433, 2000.

31. Smith LL: Mechanism of paraquat toxicity in lung and its relevance to treatment. Hum Toxicol 6: 31-36, 1987.

32. Smith LL, Lewis CP, Wyatt I and Cohen GM: The importance of epithelial uptake systems in lung toxicity. Environ Health Perspect 85: 25-30, 1990.

33. Lacerda AC, Rodrigues-Machado Mda G, Mendes PL, et al: Paraquat (PQ)-induced pulmonary fibrosis increases exercise metabolic cost, reducing aerobic performance in rats. J Toxicol Sci 34: 671-679, 2009.

34. Tung JN, Lang YD, Wang LF and Chen CM: Paraquat increases connective tissue growth factor and collagen expression via angiotensin signaling pathway in human lung fibroblasts. Toxicol In Vitro 24: 803-808, 2010.

35. Bhogal RK, Stoica CM, McGaha TL and Bona CA: Molecular aspects of regulation of collagen gene expression in fibrosis. J Clin Immunol 25: 592-603, 2005.

36. Phan SH: Biology of fibroblasts and myofibroblasts. Proc Am Thorac Soc 5: 334-337, 2008.

37. Kendall RT and Feghali-Bostwick CA: Fibroblasts in fibrosis: novel roles and mediators. Front Pharmacol 5: 123, 2014.

38. Liu X, Wu W and Chen H: Effects of CTGF gene silencing on the proliferation and myofibroblast differentiation of human lung fibroblasts. Sheng Wu Yi Xue Gong Cheng Xue Za Zhi 25: 407-412, 2008 (In Chinese). 